\title{
The complexities of the diet-microbiome relationship: advances and perspectives
}

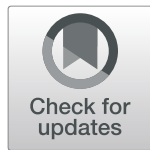

\author{
Emily R. Leeming ${ }^{1 \dagger}$, Panayiotis Louca ${ }^{1 \dagger}$, Rachel Gibson ${ }^{2}$, Cristina Menni ${ }^{1}$, Tim D. Spector ${ }^{1 *}$ and \\ Caroline I. Le Roy ${ }^{1 *}$ (iD
}

\begin{abstract}
Personalised dietary modulation of the gut microbiota may be key to disease management. Current investigations provide a broad understanding of the impact of diet on the composition and activity of the gut microbiota, yet detailed knowledge in applying diet as an actionable tool remains limited. Further to the relative novelty of the field, approaches are yet to be standardised and extremely heterogeneous research outcomes have ensued. This may be related to confounders associated with complexities in capturing an accurate representation of both diet and the gut microbiota. This review discusses the intricacies and current methodologies of diet-microbial relations, the implications and limitations of these investigative approaches, and future considerations that may assist in accelerating applications. New investigations should consider improved collection of dietary data, further characterisation of mechanistic interactions, and an increased focus on -omic technologies such as metabolomics to describe the bacterial and metabolic activity of food degradation, together with its crosstalk with the host. Furthermore, clinical evidence with health outcomes is required before therapeutic dietary strategies for microbial amelioration can be made. The potential to reach detailed understanding of diet-microbiota relations may depend on re-evaluation, progression, and unification of research methodologies, which consider the complexities of these interactions.
\end{abstract}

Keywords: Personalised nutrition, Gut microbiome, Diet, Research methods

\section{Background}

The field of microbiome research has progressed rapidly in recent years, driven by technological advances and reduced costs of analysis [1]. Significant insights have been made into the nature of microbial communities and their impact on host health [1]. However, there are inherent challenges and complexities in defining the boundary of a 'healthy' microbiome landscape [2]. Microbial signatures are highly individual and multidimensional [3] with multiple landscapes likely to be considered healthful depending on the context [4]. In a study by Ghosh et al., researchers investigated the

\footnotetext{
* Correspondence: tim.spector@kcl.ac.uk; caroline.le_roy@kcl.ac.uk

†Emily R. Leeming and Panayiotis Louca contributed equally to this work.

'The Department of Twin Research, St Thomas' Hospital, King's College

London, 3-4th Floor South Wing Block D, Westminster Bridge Road, London SE1 7EH, UK

Full list of author information is available at the end of the article
}

impact of a 1-year Mediterranean diet intervention on the gut microbiota and frailty [5]. The authors remind us of the Anna Karenina principle which posits that healthy individuals typically display microbiomes more similar to one another, while those of unhealthy individuals are each aberrant in their own way $[5,6]$. The involvement of the microbiome in an extensive number of diseases suggests the need for its incorporation into contemporary medicine for an improved understanding of disease pathogenesis and pathology [7]. A clear link exists between loss of keystone taxa that drive microbiome structure and function (such as Faecalibacterium prausnitzii [8]), and various disease states [9]. However, one of the biggest challenges in microbiome research is discerning association from causation [10]. To date, there is limited evidence to support causation in humans, predominantly due to limitations in accurately manipulating

(c) The Author(s). 2021 Open Access This article is licensed under a Creative Commons Attribution 4.0 International License, which permits use, sharing, adaptation, distribution and reproduction in any medium or format, as long as you give appropriate credit to the original author(s) and the source, provide a link to the Creative Commons licence, and indicate if changes were made. The images or other third party material in this article are included in the article's Creative Commons licence, unless indicated otherwise in a credit line to the material. If material is not included in the article's Creative Commons licence and your intended use is not permitted by statutory regulation or exceeds the permitted use, you will need to obtain permission directly from the copyright holder. To view a copy of this licence, visit http://creativecommons.org/licenses/by/4.0/. The Creative Commons Public Domain Dedication waiver (http://creativecommons.org/publicdomain/zero/1.0/) applies to the data made available in this article, unless otherwise stated in a credit line to the data. 
the human microbiome [11]. Methods such as faecal microbiome transplants (FMT) have provided evidence that the microbiome alone can causally alter the human phenotype [12]. The ability of FMT from lean donors to reorientate host glucose metabolism is influenced by the recipient's baseline microbial profile [13]. This could be explained, in part, by species from both recipient and donors remaining durably in the gut post-FMT, demonstrating the difficulties in precisely manipulating the composition of the microbiome [14].

Besides invasive solutions, therapeutic modulation of the gut microbiota could be achieved through diet [15]. Human and animal models have highlighted the influence of diet in shaping the gut microbial community through the provision of substrates for the metabolic requirements of individual or subsets of microbial taxa [16], in addition to modulating host gut microbiota crosstalk [17]. Although diet provides one of the most promising means of selectively altering the microbiome [18], current descriptions of human dietary habits and food compositions provide a simplistic insight into a complex world that is still largely unmapped [19]. Homogenous outcomes within the nutrition field are stymied by several factors. An accurate description of dietary intake is fundamental to health and nutrition research, yet capturing dietary exposure is challenging [20]. As per the microbiome, one's diet is often composed of a multitude of components that are poorly characterised individually and rarely investigated in combination or as a food matrix structure [15, 21]. While current investigations into the diet-microbial relationship have provided a broad understanding of some of these relations [22], further progress has been restricted.

We propose this review as a readout of the current flaws in diet-microbiome studies while proposing points of improvements that should enable the furthering of current knowledge in this field. Together, this should pave the way towards a global improvement of population health. Firstly, we describe the gut microbiome as a complex ecosystem with multiple interactions, secondly, we discuss the intricacies of dietary investigations, and thirdly, we consider the importance of combining these two fields when researching diet-microbe relations. For the scope of this review, we refer to the microbiome as an ecosystem which incorporates all microorganisms together with the metabolites and other components of the gut environment as defined by Marchesi and Ravel [23]. However, the predominant focus of the research within this field has been towards investigating bacteria and their interactions.

\section{The gut microbiome a complex ecosystem Multiple intricate interactions}

While phyla and functional pathways are widespread within the population (encountered in over $50 \%$ of individuals), species tend to be more subject-specific with, on average, two unrelated individuals sharing approximately $43 \%$ of species [24-26]. Functional equivalence can be explained by the notion of niches with multiple species interacting in a competitive or synergistic nature [27]. Hence, past work has shown that manipulation of a single species can prove difficult [25], while the systemwide influence of the microbial community can be achieved $[28,29]$. A number of studies reported the involvement of microbes in an orchestrated maintenance of host homeostasis. Microbial networks of species and metabolic products, for example, act on both microbes and host cell gene expression selectively dictating cellular productivity [7], emphasising the urgent development of tools that can capture the full complexity of these interactions [30, 31].

Microbial species observed within the modern gut may have evolved through ecological adaptations of host-microbe interactions to ensure microbial stability in response to periods of limited nutritional availability [7]. Even so, the gut microbiome is not a static community. The complexity of studying the microbial ecosystem is deepened by its temporal dynamics, shifting diurnally, seasonally, and in constant flux [32, 33] requiring longitudinal investigations [34]. The high rate of strain-level turnover may allow for microbial evolution to impact certain species' long-term persistence and colonisation in the gut [35]. In vitro and model organisms have assisted our current understanding of these dynamics, however through the lens of a reduced or simplified microbial ecosystem [36]. Mathematical models should be developed combining large human datasets together with in vitro and in vivo modelling in order to incorporate these dynamics (Fig. 1) [41]. Likewise, further emphasis must be made to interpret temporal dynamics through longitudinal sampling, with a limited number of longitudinal microbiome studies to date [27].

Mechanistic studies are difficult to implement in humans due to immense genetic and lifestyle heterogeneity together with ethical limitations [42]. Consequently, the functional contribution of the gut microbiome to human physiology remains largely unexplored [43]. Current knowledge stems from animal models, in vitro and in vivo assays, and is complemented by populationbased studies [42]. In this way, Suez et al. displayed that non-caloric artificial sweeteners (NAS) induced glucose intolerance in both mice and humans via modulation of the gut microbial community [44]. Functional analysis of the saccharin-associated metagenome suggests a number of enriched pathways in heterocyclic compound metabolism, with a proliferation of certain taxa possibly linked with their capacity to harness saccharin as an energy source [44]. Several studies have explored mechanistic modelling directly in humans. Sanna et al. used 


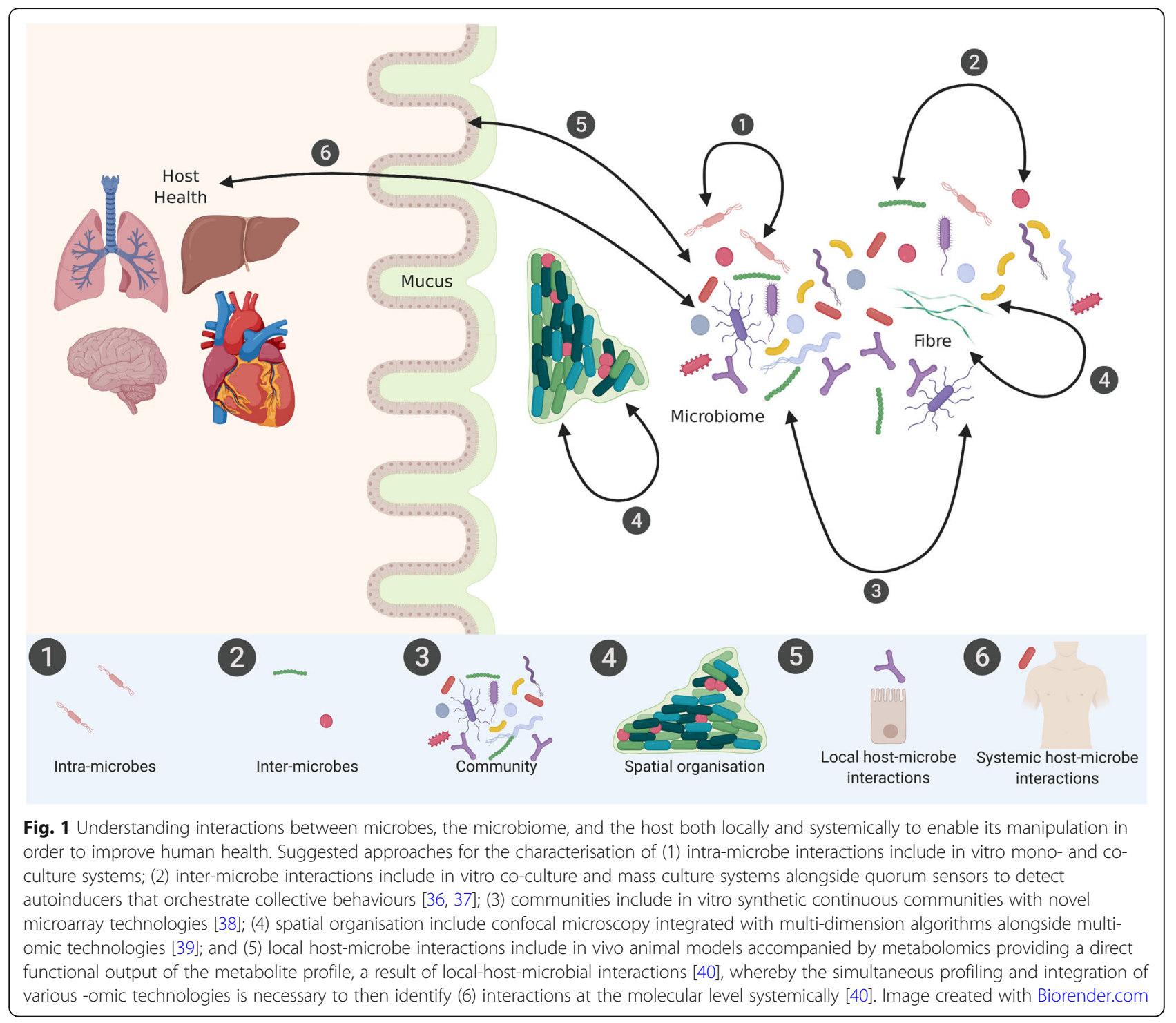

bidirectional Mendelian randomisation (MR) analyses to assess causality in a cohort of 1539 individuals from the LifeLines cohort [45]. An increase in microbial butyrate production driven by host genetics was reported to be associated with an ameliorated insulin response to an oral glucose-tolerance challenge. Likewise, abnormal production or absorption of propionate was shown to be causally related with an increased risk of type II diabetes [45]. Characterising the functional differences of the microbial taxa is fundamental in understanding their impact on human physiology [43]. Functional -omics and FMT [46] have demonstrated their potential in assisting in the identification of functional gut microbial traits [43], in conjunction with other more traditional measures. Detailed theoretical simulations to characterise the functional difference of specific microbiological ecosystems are also thought to be feasible, such as Larsen and Claassen's work which support the mechanistic link between alpha-diversity and health [47].

The spatial organisation of microbial communities is critical to understanding microbial signalling and metabolic interactions at a micron-scale but is yet relatively uncharted territory (Fig. 1) [48, 49]. Within this biogeography, microbial taxa tend to be localised according to their functional niche, for example, anaerobic taxa typically residing to the interior and consumers and producers of a metabolite found to be within equidistance of each other $[50,51]$. The availability of relevant substrates within the gut is also expected to drive this spatial organisation. While multi-omic techniques such as metagenomics, metabolomics, transcriptomics, and proteomics provide key tools in investigating the intricate crosstalk within the microbial community and between microbes and host, these techniques tend to be 
applied to homogenised samples [49] with spatial heterogeneity typically neglected. The use of confocal microscopy on biopsy samples enables the identification of single cells, a specific challenge in the dense cellular environment within the lower intestine [49]. Several tools have been developed to facilitate semi-automated curation of cell boundaries, which can be challenging due to close bacterial contact, or pixel-based quantitative measures for large-scale measurements [49]. Integration of spatial organisation within multi-dimensional algorithms, in conjunction with -omic technology, may assist in advancing the study of the organisation and dynamics of the microbial community and its relationship with host health. Retention of the structure by dissecting and spreading out the sample on a slide may aid in revealing the spatial organisation. With this method, investigations of the oral microbiome using dental plaque allowed for characterisation of the microbiota as highly structured with multi-genus consortia [50]. The gold standard approach is whole-stool homogenised sampling, although implementation of this can be impractical. Whether to collect complete or partial stool samples or biopsy specimens should be considered by the investigators and is dependent on feasibility, costs, patient cooperation, and downstream analysis [52].

Finally, the typical faecal sample represents the final point of a developing and maturing ecosystem through the gastrointestinal tract [53]. With varying gut transit times between individuals, the collection of dietary data and its corresponding stool sample can be fraught with inconsistencies [54]. Faecal consistency, a proxy for transit time, has been identified as a major co-variate of microbial structure. This suggests that transit time data should be included in future microbiome investigations and considered when capturing dietary information [55]. While there are several validated measures of transit time, including scintigraphy and radiopaque markers, many are expensive with high participant burden [56]. Other cheaper scalable measures include the blue dye method, faecal consistency, and frequency [57].

\section{Optimising microbial data collection, storage, and analysis}

Microbiome data processing pipeline, collection, storage, and analysis of samples are particularly vulnerable to significant error $[54,58]$ that contribute to high variability in research outcomes [59]. Collection methods include variable storage temperatures, freeze-thaw cycles, lysis conditions, and physical perturbations [54]. While a detailed approach is required, protocols that are perceived as too arduous can induce attrition bias in and of themselves [54]. Numerous techniques and differing protocols have been suggested with a verified gold standard approach yet to be established [52].
Traditional culture-based technologies that have been used to investigate the microbial ecosystem have recently regained attention with the development of new methods enabling the culturing of an extended number of bacteria from the human gut. Though bacterial taxa including Ruminococcaceae and Faecalibacterium tend to be overrepresented by these methods [60]. Besides, only $50-60 \%$ of bacterial species present in the human gut have been observed to produce spores resistance to multiple environmental challenges [60], thereby facilitating transmission from host-to-host [61], consequently limiting the scope for FMT studies. While this is an expensive, cumbersome approach with clear methodological limitations, the majority of current knowledge within microbiome research originates from culturebased studies and has been informative in steering future directions with more progressive techniques [62].

Development of new methodologies should assist in addressing sample processing bias. For example, whole shotgun metagenomic sequencing has allowed investigators to bypass the PCR amplification used within $16 \mathrm{~s}$ rRNA sequencing related to an overestimation of certain taxa [63]. Outside of the wet lab, a wide variety of bioinformatics tools can be used to classify microbial taxa from sequencing data [64]. A number of bioinformatic tools are publicly available for quality control, sequence assembly, operational taxonomic units, functional profiling, and prediction and to determine diversity evenness and richness [65]. Resources such as PICRUSt use evolutionary modelling to predict metagenomes from $16 \mathrm{~S}$ data and a reference genome database [66] and have shown correlations between inferred and metagenomically measured content of close to 0.9 [66]. Platforms such as MGnify, a free-to-use platform for the assembly, analysis and archiving of microbial data have allowed for publicly available analysed datasets [67]; however, extensive action is required to populate these platforms, and differences between pipelines can also lead to variations in outcomes.

Improved accuracy and throughput of DNA sequencing techniques, together with multi-omic analysis and mechanistic experiments in animal models, increased our understanding on the structure and function of the microbiome in health and disease [42]. Several methodologies are required to further characterise how microbial functionality may relate to health and disease [68]. These include but are not limited to (i) the development and application of molecular and cellular highthroughput measurements; (ii) experimental models and human studies of direct molecular effects [43], for example, the use of germ-free mice can provide insights into disease causality [69]; and (iii) the incorporation of transcriptomics and epigenetic data into the gut metagenomic profile. These allow us to understand how a shift 
in microbiome composition can modify pathways involved in disease pathogenesis. For instance, microbiotadependent histone modification has emerged as a molecular mechanism involved in tumour suppression, although findings are currently non-conclusive [34]. These steps will facilitate research in shifting away from inferences and towards a more causative understanding of the relationship between the microbiome and the pathogenesis of disease states.

\section{Capturing dietary diversity and food interactions Food is rarely consumed on its own and contains numerous compounds}

The complexity of the human gut microbiome is largely mirrored by that of diet [4]. While we have a broad understanding of the impact of diet on the gut microbiota, formulating meaningful targeted dietary strategies remains a key challenge [70]. Foods are rarely consumed alone, and the number of available combinations is incalculable, although some may be more recurrent than others [19]. Diet is a highly individualised, multifaceted and changing measure, yet specifically linked to the geographical and cultural context, restraining research generalisation [71, 72]. Variable nutrition content within the same food item related to climate, soil type, and season also contribute confounding factors and limitations [73, 74]. Food composition values within food composition databases are typically obtained from laboratory analyses; however, due to the high procedural costs, many values are estimated based on conversion factors or as a ratio of similar food types [75]. There is limited potential to consider the biochemical digestibility, absorption and subsequent bioavailability of substrates for microbial communities as a time-dependent process and one which is highly variable between individuals [76]. No method of collecting dietary data is totally devoid of error, and the efficacy of each is dependent on the scenario. The guidance of research dietitians in diet investigations is strongly recommended, with inconsistent findings as a result of suboptimal use of dietary assessments. While numerous significant or strong observations have been determined by epidemiological studies, these have not always been supported by the outcomes of randomised controlled trials [77]. Failure to confirm a dietary effect may be due to a magnitude of cumulative biases [78], in conjunction with a small effect size, amongst others, rather than a lack of validity.

While a shift towards bigger datasets is undoubtedly required, this cannot be considered a 'catch-all' solution. With dietary bioactive compounds acting synergistically, and present within a multitude of food sources, almost all nutritional variables correlate between each other and health outcomes, particularly evident in large datasets [79]. Other factors such as eating behaviours, eating times, nutrient provenance, habitual diet, and other social and behavioural factors are not currently addressed within the majority of diet-microbiome investigations, yet all play a role in mediating host health $[15,80]$. A move from simplistic reductionist strategies towards multi-faceted approaches is required.

Advancing nutritional research techniques has not progressed at the same pace as the rapid development of microbial investigations in the last decade. To improve understanding of diet-microbial relations, drastic progress is required to further our ability to characterise diet beyond the established macro and micronutrients. Emphasis on the importance of accurate dietary data methodologies and application of techniques typically applied within other scientific research fields, such as machine learning, may assist. While the nutritional community includes outstanding scientists, a large number of dietary research are undertaken by investigators in other fields, without the input of dietitians, nutritionists, or scientists trained in nutritional epidemiology. This may limit the quality of dietary data collection, processing, analysis, and reporting. For diet-microbial investigations, the involvement of a trained nutrition research professional should undoubtedly improve research outcomes and aid in the elucidation of some of the intricacies within these relations.

\section{Dietary data collection, processing, and analysis for microbiome studies Study design}

In designing study protocols for diet-microbiome investigations, collaborations between dietitians and/or nutritionists and microbiologists, epidemiologists, and biostatisticians are essential in order to capture a broad spectrum of accurate data. Establishing the cause and effect of diet has been acknowledged as challenging [81]. Nutritional epidemiology typically identifies dietary components that modify health risk, which can then be tested within a clinical trial [82]. Controlled feeding studies are considered to be robust in determining cause-and-effect relationships between diet and physiological health outcomes, as they facilitate deep phenotypical analysis [81]. Nevertheless, only a small fraction of studies are of an experimental study design within the diet-microbial field. Interventions investigating diet and health require a large amount of participant burden, substantial time and financial costs, and a high level of participant commitment [83]. Habitual diet is acknowledged to play a strong role in shaping the microbial ecology through the daily provision of substrates [15]. The collection of habitual dietary data may be essential regardless of the investigative format and should be incorporated into experimental study designs (Fig. 2). Previous work suggested that gut microbial communities 
can be clustered into typical 'enterotypes', defined as densely populated multi-dimensional areas in the gut microbial community. These 'enterotypes' could be used as a way to stratify samples to reduce complexity [84] and previously have been linked to cardiometabolic risk [85], and a differential response to T2D treatment [86]. Microbial and metabolic phenotypes exhibit enterotypespecific links, emphasising the importance of enterotype stratification in investigating metabolic responses to diet [87]. Multi-centre studies allow for the investigation of enterotypes in wider population groups. These acknowledge the influence of geographical, ethnic, and cultural influences on the microbiome and diet amongst others. Investigations of diet-microbe relations, particularly multi-centre randomised control trials, that stratify according to enterotype profile and account for baseline habitual diet, with longitudinal sampling and health measures, may lead to increased homogeneity of outcomes. In combination, these suggestions would undoubtably assist in promoting general, and individualised or enterotype-based, diet-microbe therapeutic recommendations for the prevention or amelioration of relevant disease states (Fig. 2).

\section{Measurement}

Investigators of diet-microbiome relations often rely on food frequency questionnaires and self-reported food diaries [88]. Yet, multiple weighed 24-h dietary recalls, involving a retrospective assessment held by a trained nutrition professional or dietitian, are generally acknowledged to provide the highest accuracy in capturing dietary intake [72]. Resources such as the DIETary Assessment Tools NETwork (DIET@NET), who developed the Nutritools website [89], facilitate researchers' awareness of the strengths and weaknesses of dietary assessment methods [90, 91], summarised in Table 1. High participant burden and costs, such as interview time and data entry, limit the utility of dietary recalls for large cohorts [72], though recent progress in technological applications, such as web-, app-, and computer-based 24-h
Current

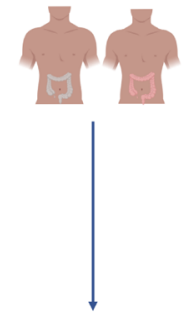

Dietary intervention

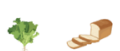

Reduced number of timepoints

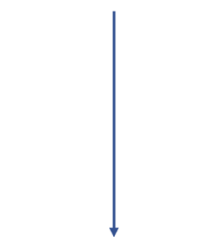

Heterogeneous outcome

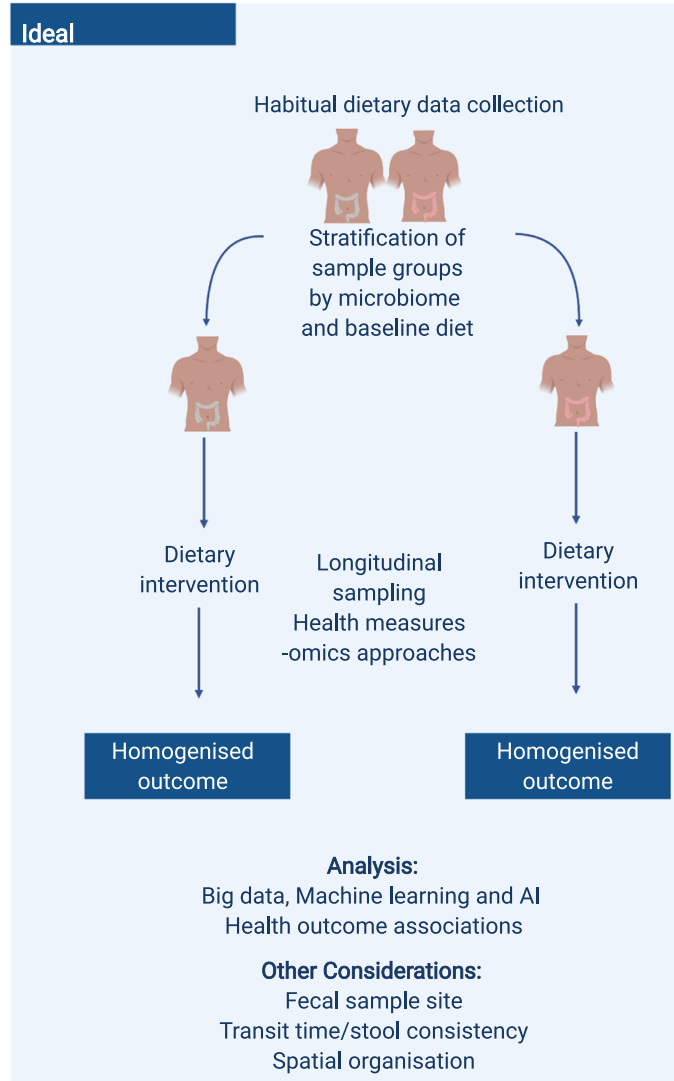

Fig. 2 Current approaches vs. ideal approaches (image modified from Leeming et al. [15]). Current microbiome-diet-host approaches carry a number of caveats which may contribute to highly heterogeneous responses, such as the individualised microbiome [15]. A new ideal approach that may allow for further elucidation of diet-microbial-host relations includes stratification by microbial signature, collection of habitual diet data, longitudinal sampling and big data, machine learning, and Al approaches in order to enhance the predictability of outcomes in response to the dietary intervention. Image created with Biorender.com 
Table 1 Advantages and disadvantage of dietary assessment methods

Dietary assessment tool Strengths Weaknesses

\section{Retrospective}

Dietary recalls - short-term method, where foods and drinks consumed are recalled.

Food frequency questionnaire-retrospective methods recording frequencies of common foods over a period of time (weeks, months, years). Can be qualitative (frequency only), semiqualitative (estimated portion size), or quantitative (portion size queried).
- Self-reported manually or electronically.

- Can be interview led, face-to-face, by phone, or online.

- Typically, recall past $24 \mathrm{~h}$ but can be employed to recall longer durations/instances.

- Multiple 24-h recalls can estimate habitual intakes.

- Facilitates collection of extra information (meal timing, frequency, and location).

- Flexibility of collected data is applicable to diverse research questions and analytical methods.

- Limited literacy skills and cultural differences can be overcome using an interviewer.

- Moderate participant burden and high compliance rates.

- A skilled interviewer using multi-pass methods can prompt information, increasing accuracy.

- Self-reported manually or electronically or interviewer led.

- Useful for estimating long-term intakes retrospectively.

- Low cost and participant burden, higher completion rate, applicable to large population studies.

- Comprehensive questionnaires can estimate total nutrient intake if the portion size is prompted.

- Can utilise short questionnaires specific to foods or nutrients pertinent to the research question.

- Analysis is typically less burdensome on researchers.
- Habitual intake can change during the recall period. Overcome if participants are not forewarned.

- Limited accuracy when recalling distant periods.

- Unsuitable for subjects with memory disorders or elderly.

- Items often omitted and incorrect items can be recalled.

- One 24-h recall has limited accuracy, typically underestimating intake and overlooks day-to-day variation.

- Moderate-to-high burden when analysing, requiring standardised protocols.

- Expensive to employ face-to-face interviews in studies with large samples.

- Reliance on subjects' ability to remember portion size.

- Arduous for participants if $>100$ food items are queried.

- Limits comparisons across cultures/countries unless comparable diets.

- Typically, shorter questionnaires have less reliability and accuracy of intake.

- Relies on participants' memory, literacy, and numeracy skills. Longer periods of time reduces the accuracy of intakes.

- Requires a proxy for accurate reporting in children.

- Prone to misreporting, particularly with

longer questionnaires.

- Finite list of items included in the questionnaire

- Expensive software required to convert frequencies to nutrients.

\section{Prospective}

Food diaries - prospective methods where details of everything consumed is logged over several days. Portions can be either estimated by the subject or via photographical evidence or weighed by the subject or research assistant at the time of consumption.

Dietary checklist-prospective short-term method where specified foodstuffs are ticked from a checklist over a number of days. Can include frequencies or portion sizes. Typically used as a screening tool. Shares many strengths and weaknesses of FFQs.
- Provides detailed depiction of foods and drinks - Not applicable to retrospective studies. consumed, including portions.

- Generates good estimates of short-term dietary intake, if conducted thoroughly.

- Facilitates collection of contextual data (meal timing, location, satiety levels).

- Not influenced by subjects' memory if recorded prospectively.

- Weighed provides more accurate quantitative intake, can also include ingredients and food waste.

- Can be conducted via digitally or manually.

- Prompts can promote the inclusion of specific foods, nutrients or occasions, pertinent to the research question and limit misreporting.

- Reasonably cost-effective.

- Accuracy increases with standardised protocols and analysis.

- Useful for estimating dietary patterns over short periods.

- Low cost.

- Low participant and researcher burden.

- Relatively simple coding.
- High participant burden, particularly over longer durations, adding to the high participant burden of microbiome research.

- Costly in time and resources for coding and analysis.

- Compliance rate reduces as the duration of recording increases.

- Requires sufficient literacy and numeracy skills of subject/proxy.

- Heavy reliance on subjects' perception of portions (can be improved with photographs)

- Relies on trust that the diary is complete at the time of consumption and not as a recall.

- Generally, very short, cannot determine total intakes. This is of concern for microbial research as determination of effects are limited.

- Cross-cultural/cross-country comparisons are limited unless diets are comparable.

- Restricted to items that are listed in the instrument. 
Table 1 Advantages and disadvantage of dietary assessment methods (Continued)

\begin{tabular}{|c|c|c|}
\hline Dietary assessment tool & Strengths & Weaknesses \\
\hline \multicolumn{3}{|l|}{ Retrospective and prospective } \\
\hline $\begin{array}{l}\text { Diet histories-combination of multiple } \\
\text { methods, typically } 24-\mathrm{h} \text { recalls, food frequency } \\
\text { questionnaires, and food diaries. More applicable } \\
\text { in clinical settings by experienced dieticians to } \\
\text { generate an in-depth analysis at an individual } \\
\text { level. }\end{array}$ & $\begin{array}{l}\text { - Long periods > } 1 \text { month can determine } \\
\text { habitual intake. } \\
\text { - Combinations of methods is ideally suited to } \\
\text { capture accurate dietary intake during a period } \\
\text { of interest surrounding faecal matter collection. } \\
\text { - Facilitates assessment of meal patterns and } \\
\text { food preparation. } \\
\text { - Typically uses automated tools that have been } \\
\text { adapted for self-administration. }\end{array}$ & $\begin{array}{l}\text { - No standardised protocols available. } \\
\text { - Meal based approaches is not suitable for } \\
\text { individuals with irregular eating patterns. } \\
\text { - High participant and researcher burden. } \\
\text { - Requires complex analytical methods. } \\
\text { - Expensive, as requires experienced } \\
\text { interviewer and researcher to code data. }\end{array}$ \\
\hline $\begin{array}{l}\text { Novel technologies_collect and process } \\
\text { dietary data using wearable hardware (such as } \\
\text { sensors) and software (such as web-based pro- } \\
\text { grammes and mobile apps based on traditional } \\
\text { dietary assessment tools). Many have close } \\
\text { agreement to traditional methods, yet notice- } \\
\text { able differences persist when comparing against } \\
\text { the gold standard, doubly labelled water tech- } \\
\text { niques [92]. }\end{array}$ & $\begin{array}{l}\text { - Facilitates real-time data entry and results irre- } \\
\text { spective of location. } \\
\text { - Enhanced portion size quantitation and food } \\
\text { waste estimating using digitally captured } \\
\text { photos. } \\
\text { - Reduces participant burden and increases } \\
\text { motivation (dependent on participants' } \\
\text { technological ability). } \\
\text { - Facilitates easier prompting to reduce mis- } \\
\text { recording. } \\
\text { - Automation of web-based recording reduces } \\
\text { the burden on researchers and interviewers. }\end{array}$ & $\begin{array}{l}\text { - Due to novelty, no validation performed to } \\
\text { determine the quality of the technology. } \\
\text { - Prone to similar measurement errors as } \\
\text { traditional assessment tools. } \\
\text { - Potential security risk using a web-based } \\
\text { computer or mobile-technologies. } \\
\text { - Requires participant education/training if } \\
\text { the tool is not intuitive. } \\
\text { - Potential high initial costs of specialist } \\
\text { equipment and software. }\end{array}$ \\
\hline
\end{tabular}

This table is adapted from Nutritools [89]

dietary recall tools, may mitigate some of these limitations [93]. A minimum of four to eight repeated 24-h dietary recalls have been previously recommended to accurately characterise habitual dietary intake [94, 95]. Typically, food frequency questionnaires (FFQs) are employed in nutrition research for large population studies as a cost-effective tool for assessing habitual diet [88].

\section{Bias, error, and further limitations}

Besides collection methodology, the majority of nutrition research investigates diet as a limited series of macro and micronutrients [96] covered by food composition databases [19]. Dietary mediators of gut microbial action may not be described, overlooking the impact of $>26$, 000 biochemicals encapsulated within the food matrix, as well as the production, preparation, and consumption of foods with the potential passage of environmental and food-borne microbial communities [16, 19]. Zhang and Li recently demonstrated that consuming cooked foods drastically reduced microbial diversity in comparison with consuming non-thermally processed foods in an animal model [97]. Moving towards detailed descriptions of foods is required, including food processing, cooking methods, and mealtime [76, 98] together with rhythmicity of nutritional responses of nutrient sensing and cellular decision-making [71]. Certain biochemicals have been observed to mitigate or exacerbate the health effects of others present in other foods [19]. For instance, trimethylamine N-oxide (TMAO), a product of trimethylamine (TMA) transformation by the liver, has been extensively associated with the negative health effects linked to red meat consumption [99]. Yet, allicin, a biochemical in garlic, blocks the microbial generation of TMA in the gut, preventing the potential adverse effects of TMAO [19]. Many other bioactive compounds found in food are separately documented elsewhere within the literature; however, extensive systematic collaboration is required for a unified database. Barabasi et al. displayed that an advanced library for garlic and cocoa can be developed by integrating machine learning into study searches for aggregation despite numerous diverse sources [19, 100]. Future efforts could utilise this technology to efficiently analyse large datasets to develop global databases for the benefit of researchers and institutes in the food domain [101]. For example, the FiberTAG project is tagging fibre types, including soluble and insoluble dietary fibre and prebiotic oligosaccharides, by measuring biomarkers related to the gut microbiota in order to aid progression in future diet-microbiome research [102].

Although epidemiological research has succeeded in identifying a link between the gut microbiome and nutrients derived from food composition databases [103], the association with specific food sources remains underexplored. Johnson et al. recently highlighted that measuring food intakes may provide increased insight into dayto-day variations in microbiota than a traditional nutrient model [55]. For example, red wine has been shown to be associated with increased microbial alpha-diversity that was not observed with other alcohols hypothesised to be related to the high polyphenol content of red wine [104]. However, the investigators were unable to confirm due to the restricted descriptions of bioactive compounds available. 
While many further limitations and biases are worthy of discussion, the final note on this subject should highlight the importance of rigorous reporting to allow for scientific reproducibility. Standardised reporting guidelines of all future research efforts should be developed following a consultative process [105]. Extensions to current guidelines, such as the STROBE-nut, have been successfully implanted [105]. While these are currently only specified within a number of nutrition journals, investigators are encouraged to incorporate the guidance offered despite this.

\section{Investigating diet-microbiome interactions}

The pliable nature of the gut microbiota composition facilitates its modulation via environmental factors, the most important of which is diet [106]. Yet, to date, unscrambling the effects of diet and the gut microbiota on host health has proved challenging; particularly considering the two are closely aligned [103]. Moreover, the presence of highly complex crosstalk between diet, microbiota, and the host has proven a major confounder
[4] with full characterisations of the complex interactions between dietary substrates and metabolites, and the crosstalk between host and microbes not yet fully explored [107].

Diet influences not only the microbial composition, but also regulates the activity of the ecosystem (and its effects on the host) without noticeable compositional alterations [108] (Fig. 3). Further investigations to identify factors that influence these three-way interactions between the host, diet and the microbiota are required. These complex and intricate relations demands the employment of a holistic approach moving beyond simple association studies [110]. While diet may at times have minimal impact on the microbial community structure, the production of dietary metabolites may differ [111]. Thousands of dietary biomolecules are present within a food matrix, many of which are unknown [19]. Identification of strains implicated in the metabolism of dietary substrates remains unclear with multiple others performing similar or the same pathways, some of which work synergistically [16]. To date, the wealth of

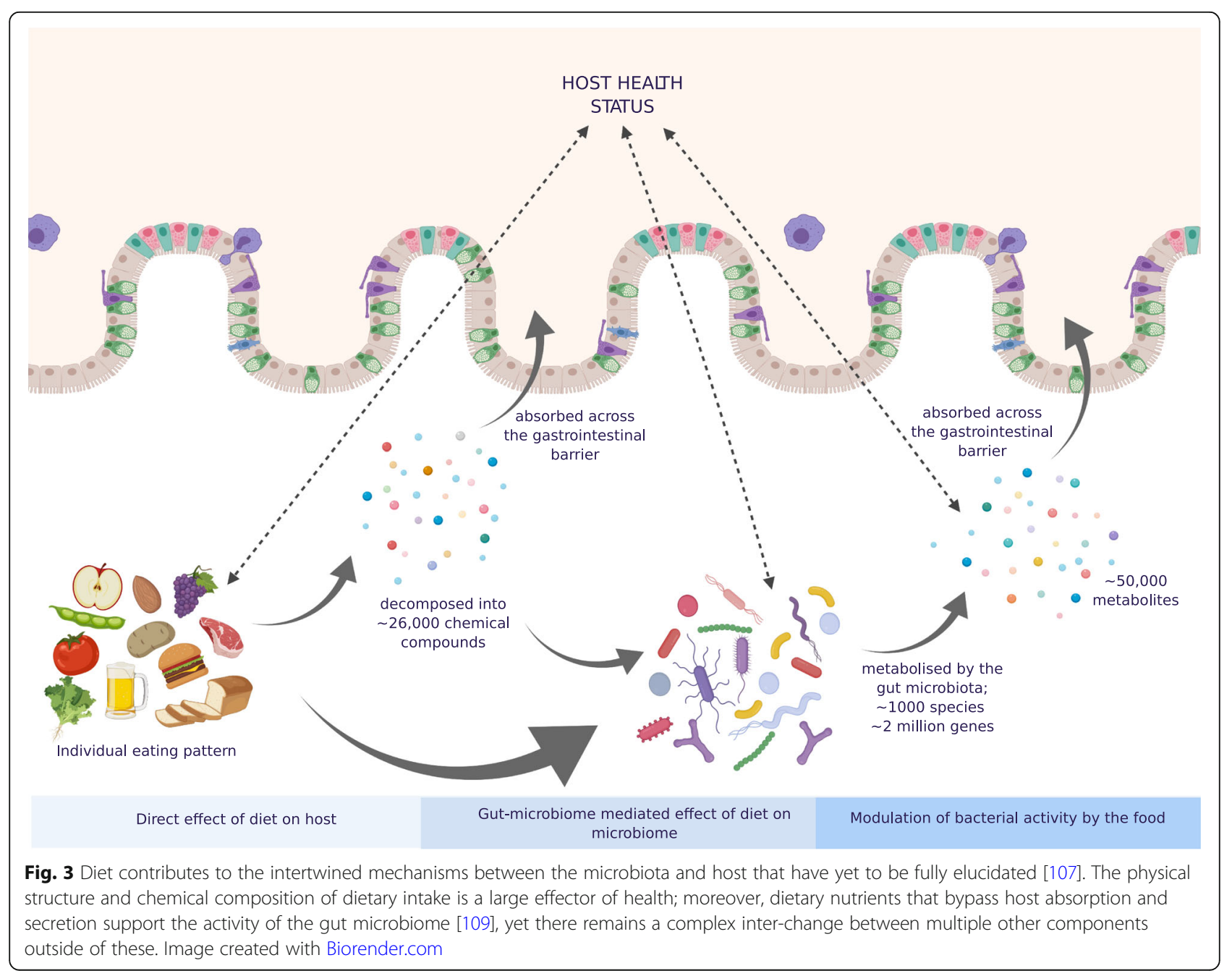


metabolic functionality studies has demonstrated the extensive reach of the gut microbiota throughout the metabolic system of gnotobiotic [112], antibiotic-treated animals [113, 114], and also some human studies [115]. Recent advances in mathematical models to capture key aspects of the gut microbiome and its hosts' physiological response facilitate the generation of hypotheses that can later be experimentally validated [115]. The future inclusion of data points in artificial intelligence (AI) models beyond the typical may also further assist in expanding our understanding of diet-microbiome relations, for example, the functional genomic analyses of carbohydrate utilisation of strains and species and the cross-feeding of fermentation products and vitamins unidirectionally or bidirectionally $[116,117]$. For instance, the growth of many butyrate-producing gut bacteria, such as F. prausnitzii, S. variabile, and Roseburia, has been shown to be auxotrophic for B vitamins, and therefore rely on exogenous sources [117], with links between increased consumption of B vitamins and abundance of taxa [118]. By including such data, a greater understanding of diet-based approaches to modulate beneficial microbes and improve health may be modelled [117, 119].

Reconceptualization of the current approaches towards big data technical methodologies and improved study design may assist in further characterisation of the dietmicrobial landscape [120]. Technological advances in high-throughput -omic technologies have greatly improved the accessibility to functional information surrounding the microbiome [49]. Such studies are indispensable for the progression of the field alongside the increased focus on developing comprehensive and reproducible workflows and improved choice of methods and scientific rigour in the conduct of the study [59]. Integrating the fields of microbiology, genetics, epigenetics, metabolomics, proteomics, and nutrition, we can consolidate our understanding of techniques, thereby develop investigations which may capture a richer and more coherent picture [121]. For example, in a 2019 study, investigators explored diet-microbiome relations and their individual impact on visceral fat mass (VFM). The pair-wise association and conditional analyses, together with machine learning approaches, enabled to both estimate and separate the effects of diet and the gut microbial community on host VFM [103]. Additionally, the integration of multiple fields assists in overcoming some of the limitations of individual technologies by looking at a broader picture of disease networks rather than, for example, compounds in isolation [122]. Furthermore, the emergence of novel visualisation tools such as bio-orthogonal click chemistry labelling [123] and optical windows for real-time tracking has shown potential but has yet to be fully applied [49].
Consideration must be made as to how to best integrate quantitative imaging techniques with the quantitative pipeline to advance diagnostics, improve population health through disease prevention and management [49].

Longitudinal large multi-centre studies are required which employ standardised protocols for the collection of validated biomarkers of health for phenotyping, subject demographics, dietary information, biological samples, laboratory processing, genetic analyses, and data analysis and manipulation [3]. Generation of substantial data pools could be overcome by integration of the mechanistic, hypothesis-driven approach with machine learning AI [124]. Machine learning methods to identify microbiota characteristics associated with host phenotypes of interest can be categorised into two types, supervised and unsupervised learning [125]. Supervised learning can be useful when aiming to predict a health outcome or a phenotype based on microbiome profiles. It also enables the formation of a prediction model based on the multitudes of microbial taxa, enabling a view of the ecosystem rather than organisms in isolation. Unsupervised learning can also assist in identifying patterns within the ecosystem as well as within a population, as demonstrated by the concept of enterotypes [125]. Although currently in its infancy within the microbiome field, AI-based recommendation systems (RS) have shown promise [126]. By integrating blood parameters, dietary patterns, anthropometrics, physical activity, and gut microbiota into a RS, Zeevi et al. were able to predict glycaemic response to meals [127]. The researchers successfully manipulated dietary intake to alter the gut microbiome, enabling them to reduce host postprandial glucose response [127]. However, RS are limited by our current incomplete understanding of microbial metabolic pathways, microbial community, and definition of a healthy microbiome.

Large databanks typically include limited phenotypes to limit researcher burden and cost, whereas typically small finite samples facilitate more in-depth phenotyping [128]. Maximising phenotypic trait data within a substantial sample size allows for cohorts to be sufficiently powered to discover and replicate associations [129]. Meta-analysis techniques can then be used to pool samples or to combine with clinical trial results in order to detect 'true' signals and to reduce false-positive rates, strengthening the findings by demonstrating reproducibility [130]. By providing data from varying perspectives, researchers are able to answer a diverse array of scientific questions, whereby findings are more generalisable [128]. For example, AI technologies, such as those mentioned above, have been shown to outperform humans in predicting patient re-admission following congestive heart failure [131], though these technologies alone cannot provide translatable information for human health, 
requiring integration of $\mathrm{AI}$, interventions, and mechanistic studies.

\section{Conclusions and outlook}

To advance our understanding of the role of dietmicrobiota interactions on human health and disease, it is crucial to step back and re-evaluate current approaches. Standardisation and optimisation of methodologies may assist in capturing the complex spectrum of these relations. To inform dietary strategies for the prevention and amelioration of chronic metabolic disease states, we first need to ensure intrinsic data is sufficient and relevant, in a manner that considers the deeply individual aspects of both diet and microbiome. Characterisation of the natural intricacies of the ecosystem and the interactions existing between its multiple members at various levels of complexity remains critical. Both micro- and macro-scale influences that drive microbial variation should be considered, from spatial organisation to transmission amongst hosts and between the host and the environment. A comprehensive, multidisciplinary research agenda is required to accurately describe the gut microbial composition and function. Individual and combined complexities of both microbial research and nutrition research demand reconsideration of standard approaches, with a push towards gold standard protocols, further emphasis on the use of randomised control trials, and mechanistic studies, and analysis techniques that include big data, multi-omics, and machine learning approaches. Without multifactorial approaches towards investigations of the diverse aspects of the microbiome, diet, and diet-microbiome relations, we will be limited in our progression towards therapeutic interventions on a personalised or population level. While the path ahead may be unclear in how we may reach these targeted strategies to improve host health, the approaches outlined within this review may assist in a collaborative move forward.

\section{Abbreviations}

FMT: Faecal microbiome transplantation; TMAO: Trimethylamine N-oxide; TMA: Trimethylamine; VFM: Visceral fat mass; RS: Recommendation systems; Al: Artificial intelligence

\section{Acknowledgements}

Not applicable

\section{Authors' contributions}

C.I.L and T.D.S were responsible for the conception. E.R.L and P. L drafted the work. E.R.L, P. L, C.I.L, C. M, and R. G substantively revised the manuscript. All authors read and approved the final manuscript.

\section{Funding}

TwinsUK is funded by the Wellcome Trust, Medical Research Council, European Union, The CDRF, the Denise Coates Foundation, the National Institute of Health (NIH), the National Institute for Health Research (NIHR)funded BioResource, DiabetesUK, and Clinical Research Facility and Biomedical Research Centre based at Guy's and St Thomas' NHS Foundation Trust in partnership with King's College London. Cristina Menni is funded by the MRC Aim-Hy project grant and Chronic Disease Research Foundation. E.R.L and P.L are funded by the Chronic Disease Research Foundation.

Availability of data and materials

Not applicable

Ethics approval and consent to participate

Not applicable

Consent for publication

Not applicable

Competing interests

T.D.S is the scientific founder of Zoe Global Ltd. E.R.L consults for Zoe Global Ltd. The remaining authors declare that they have no competing interests.

\section{Author details}

'The Department of Twin Research, St Thomas' Hospital, King's College London, 3-4th Floor South Wing Block D, Westminster Bridge Road, London SE1 7EH, UK. ²Department of Nutritional Sciences, King's College London, Franklin-Wilkins Building, 150 Stamford Street, London SE1 9NH, UK.

Received: 30 March 2020 Accepted: 25 November 2020

Published online: 20 January 2021

References

1. Cullen CM, Aneja KK, Beyhan S, Cho CE, Woloszynek S, Convertino M, et al. Emerging priorities for microbiome research. Front Microbiol. 2020;11:136.

2. Gupta VK, Kim M, Bakshi U, Cunningham KY, Davis JM, Lazaridis KN, et al. A predictive index for health status using species-level gut microbiome profiling. Nat Commun. 2020;11(1):4635

3. McBurney Ml, Davis C, Fraser CM, Schneeman BO, Huttenhower C, Verbeke $\mathrm{K}$, et al. Establishing what constitutes a healthy human gut microbiome: state of the science, regulatory considerations, and future directions. J Nutr. 2019;149(11):1882-95.

4. Zmora N, Suez J, Elinav E. You are what you eat: diet, health and the gut microbiota. Nat Rev Gastroenterol Hepatol. 2019;16(1):35-56.

5. Ghosh TS, Rampelli S, Jeffery IB, Santoro A, Neto M, Capri M, et al. Mediterranean diet intervention alters the gut microbiome in older people reducing frailty and improving health status: the NU-AGE 1-year dietary intervention across five European countries. Gut. 2020; gutjnl-2019-319654

6. Zaneveld JR, McMinds R, Vega TR. Stress and stability: applying the Anna Karenina principle to animal microbiomes. Nat Microbiol. 2017:2(9):17121.

7. Gilbert JA, Lynch SV. Community ecology as a framework for human microbiome research. Nat Med. 2019:25(6):884-9.

8. Ferreira-Halder CV, Faria AVS, Andrade SS. Action and function of Faecalibacterium prausnitzii in health and disease. Best Pract Res Clin Gastroenterol. 2017;31(6):643-8.

9. Banerjee S, Schlaeppi K, van der Heijden MGA. Keystone taxa as drivers of microbiome structure and functioning. Nat Rev Microbiol. 2018;16(9):567-76

10. Shang Q. From correlation to causation: the missing point in the study of functional foods and gut microbiota. J Funct Foods. 2019;61:103466.

11. Méndez-García C, Barbas C, Ferrer M, Rojo D. Complementary methodologies to investigate human gut microbiota in host health, working towards integrative systems biology. J Bacteriol. 2018;200(3): e00376-17.

12. de Groot PF, Frissen MN, de Clercq NC, Nieuwdorp M. Fecal microbiota transplantation in metabolic syndrome: history, present and future. Gut Microbes. 2017:8(3):253-67.

13. Kootte RS, Levin E, Salojärvi J, Smits LP, Hartstra AV, Udayappan SD, et al. Improvement of insulin sensitivity after lean donor feces in metabolic syndrome is driven by baseline intestinal microbiota composition. Cell Metab. 2017:26(4):611-9.e6.

14. Li SS, Zhu A, Benes V, Costea PI, Hercog R, Hildebrand F, et al. Durable coexistence of donor and recipient strains after fecal microbiota transplantation. Science. 2016;352(6285):586

15. Leeming RE, Johnson JA, Spector DT, Le Roy IC. Effect of diet on the gut microbiota: rethinking intervention duration. Nutrients. 2019;11(12).

16. Dutton RJ, Turnbaugh PJ. Taking a metagenomic view of human nutrition. Curr Opin Clin Nutr Metab Care. 2012;15(5):448-54. 
17. Molinero N, Ruiz L, Sánchez B, Margolles A, Delgado S. Intestinal bacteria interplay with bile and cholesterol metabolism: implications on host physiology. Front Physiol. 2019;10:185.

18. Deehan EC, Yang C, Perez-Munoz ME, Nguyen NK, Cheng CC, Triador L, et al. Precision microbiome modulation with discrete dietary fiber structures directs short-chain fatty acid production. Cell Host Microbe. 2020;27(3):389404.

19. Barabási A-L, Menichetti G, Loscalzo J. The unmapped chemical complexity of our diet. Nature Food. 2020;1(1):33-7.

20. Willett W. Commentary: dietary diaries versus food frequency questionnaires - a case of undigestible data. Int J Epidemiol. 2001;30(2):317-9.

21. Hu FB. Dietary pattern analysis: a new direction in nutritional epidemiology. Curr Opin Lipidol. 2002;13(1):3-9.

22. Conlon MA, Bird AR. The impact of diet and lifestyle on gut microbiota and human health. Nutrients. 2014;7(1):17-44.

23. Marchesi JR, Ravel J. The vocabulary of microbiome research: a proposal. Microbiome. 2015:3(1):31.

24. Visconti A, Le Roy Cl, Rosa F, Rossi N, Martin TC, Mohney RP, et al. Interplay between the human gut microbiome and host metabolism. Nat Commun. 2019;10(1):4505.

25. Coyte KZ, Schluter J, Foster KR. The ecology of the microbiome: networks, competition, and stability. Science. 2015;350(6261):663-6.

26. Lillie EO, Patay B, Diamant J, Issell B, Topol EJ, Schork NJ. The n-of-1 clinical trial: the ultimate strategy for individualizing medicine? Personalized Med. 2011;8(2):161-73.

27. Walter J, Ley R. The human gut microbiome: ecology and recent evolutionary changes. Annu Rev Microbiol. 2011;65:411-29.

28. Sheth RU, Cabral V, Chen SP, Wang HH. Manipulating bacterial communities by in situ microbiome engineering. Trends Genet. 2016;32(4):189-200.

29. Mimee M, Citorik RJ, Lu TK. Microbiome therapeutics - advances and challenges. Adv Drug Deliv Rev. 2016;105(Pt A):44-54.

30. Dave $M$, Higgins PD, Middha S, Rioux KP. The human gut microbiome: current knowledge, challenges, and future directions. Transl Res. 2012;160(4): 246-57.

31. Rooks MG, Garrett WS. Gut microbiota, metabolites and host immunity. Nat Rev Immunol. 2016;16(6):341-52

32. Nobs SP, Tuganbaev T, Elinav E. Microbiome diurnal rhythmicity and its impact on host physiology and disease risk. EMBO Rep. 2019;20(4).

33. Koliada A, Moseiko V, Romanenko M, Piven L, Lushchak O, Kryzhanovska N, et al. Seasonal variation in gut microbiota composition: cross-sectional evidence from Ukrainian population. BMC Microbiol. 2020;20(1):100.

34. Qin Y, Wade PA. Crosstalk between the microbiome and epigenome: messages from bugs. J Biochem. 2018;163(2):105-12.

35. Priya S, Blekhman R. Population dynamics of the human gut microbiome: change is the only constant. Genome Biol. 2019;20(1):150

36. Medlock GL, Carey MA, McDuffie DG, Mundy MB, Giallourou N, Swann JR, et al. Inferring metabolic mechanisms of interaction within a defined gut microbiota. Cell Syst. 2018;7(3):245 57.e7.

37. Braga RM, Dourado MN, Araújo WL. Microbial interactions: ecology in a molecular perspective. Braz J Microbiol. 2016;47:86-98.

38. Emerson D, Agulto L, Liu H, Liu L. Identifying and characterizing bacteria in an era of genomics and proteomics. BioScience. 2008;58(10):925-36.

39. Earle Kristen A, Billings G, Sigal M, Lichtman Joshua S, Hansson Gunnar C, Elias Joshua E, et al. Quantitative imaging of gut microbiota spatial organization. Cell Host Microbe. 2015;18(4):478-88.

40. Chen L, Garmaeva S, Zhernakova A, Fu J, Wijmenga C. A system biology perspective on environment-host-microbe interactions. Hum Mol Genet. 2018;27(R2):R187-R94.

41. Xiao Y, Angulo MT, Friedman J, Waldor MK, Weiss ST, Liu Y-Y. Mapping the ecological networks of microbial communities. Nat Commun. 2017;8(1): 2042.

42. Gilbert JA, Blaser MJ, Caporaso JG, Jansson JK, Lynch SV, Knight R. Current understanding of the human microbiome. Nat Med. 2018;24(4):392-400.

43. Heintz-Buschart A, Wilmes P. Human gut microbiome: function matters. Trends Microbiol. 2018;26(7):563-74

44. Suez J, Korem T, Zilberman-Schapira G, Segal E, Elinav E. Non-caloric artificial sweeteners and the microbiome: findings and challenges. Gut Microbes. 2015;6(2):149-55

45. Sanna S, van Zuydam NR, Mahajan A, Kurilshikov A, Vich Vila A, Võsa U, et al. Causal relationships among the gut microbiome, short-chain fatty acids and metabolic diseases. Nat Genet. 2019;51(4):600-5.
46. Aron-Wisnewsky J, Clément K, Nieuwdorp M. Fecal microbiota transplantation: a future therapeutic option for obesity/diabetes? Curr Diabetes Rep. 2019;19(8):51.

47. Larsen OFA, Claassen E. The mechanistic link between health and gut microbiota diversity. Sci Rep. 2018;8(1):2183.

48. Mark Welch JL, Hasegawa Y, McNulty NP, Gordon Jl, Borisy GG. Spatial organization of a model 15-member human gut microbiota established in gnotobiotic mice. Proc Natl Acad Sci U S A. 2017;114(43):E9105-E14.

49. Tropini C, Earle KA, Huang KC, Sonnenburg JL. The gut microbiome: connecting spatial organization to function. Cell Host Microbe. 2017;21(4): 433-42.

50. Mark Welch $J$, Rossetti BJ, Rieken CW, Dewhirst FE, Borisy GG. Biogeography of a human oral microbiome at the micron scale. Proc Natl Acad Sci. 2016;113(6):E791.

51. Rivera-Chávez F, Lopez CA, Bäumler AJ. Oxygen as a driver of gut dysbiosis. Free Radic Biol Med. 2017;105:93-101.

52. Allaband C, McDonald D, Vázquez-Baeza Y, Minich JJ, Tripathi A, Brenner DA, et al. Microbiome 101: studying, analyzing, and interpreting gut microbiome data for clinicians. Clin Gastroenterol Hepatol. 2019;17(2):218-30.

53. Falony G, Vieira-Silva S, Raes J. Richness and ecosystem development across faecal snapshots of the gut microbiota. Nat Microbiol. 2018;3(5):526-8.

54. Vandeputte D, Tito RY, Vanleeuwen R, Falony G, Raes J. Practical considerations for large-scale gut microbiome studies. FEMS Microbiol Rev. 2017:41(Supp_1):S154-S67.

55. Johnson AJ, Vangay P, Al-Ghalith GA, Hillmann BM, Ward TL, Shields-Cutler $\mathrm{RR}$, et al. Daily sampling reveals personalized diet-microbiome associations in humans. Cell Host Microbe. 2019;25(6):789-802.e5.

56. Corsetti M, Costa M, Bassotti G, Bharucha AE, Borrelli O, Dinning P, et al. First translational consensus on terminology and definitions of colonic motility in animals and humans studied by manometric and other techniques. Nat Rev Gastroenterol Hepatol. 2019;16(9):559-79.

57. Compher C, Rubesin S, Kinosian B, Madaras J, Metz D. Noninvasive measurement of transit time in short bowel syndrome. JPEN J Parenter Enteral Nutr. 2007;31(3):240-5.

58. Watson E-J, Giles J, Scherer BL, Blatchford P. Human faecal collection methods demonstrate a bias in microbiome composition by cell wall structure. Sci Rep. 2019;9(1):16831.

59. Poussin C, Sierro N, Boué S, Battey J, Scotti E, Belcastro V, et al. Interrogating the microbiome: experimental and computational considerations in support of study reproducibility. Drug Discov Today. 2018;23(9):1644-57.

60. Browne HP, Forster SC, Anonye BO, Kumar N, Neville BA, Stares MD, et al. Culturing of 'unculturable' human microbiota reveals novel taxa and extensive sporulation. Nature. 2016:533(7604):543-6.

61. Swick MC, Koehler TM, Driks A. Surviving between hosts: sporulation and transmission. Microbiol Spectr. 2016;4(4)

62. Almeida A, Mitchell AL, Boland M, Forster SC, Gloor GB, Tarkowska A, et al. A new genomic blueprint of the human gut microbiota. Nature. 2019; 568(7753):499-504.

63. Hugerth LW, Andersson AF. Analysing microbial community composition through amplicon sequencing: from sampling to hypothesis testing. Front Microbiol. 2017;8:1561.

64. Mclntyre ABR, Ounit R, Afshinnekoo E, Prill RJ, Henaff E, Alexander N, et al. Comprehensive benchmarking and ensemble approaches for metagenomic classifiers. Genome Biol. 2017;18(1):182.

65. Eetemadi A, Rai N, Pereira BMP, Kim M, Schmitz H, Tagkopoulos I. The computational diet: a review of computational methods across diet, microbiome, and health. Front Microbiol. 2020;11:393.

66. Langille MGI, Zaneveld J, Caporaso JG, McDonald D, Knights D, Reyes JA, et al. Predictive functional profiling of microbial communities using $16 \mathrm{~S}$ rRNA marker gene sequences. Nat Biotechnol. 2013;31(9):814-21.

67. Wallach JD, Boyack KW, loannidis JPA. Reproducible research practices, transparency, and open access data in the biomedical literature, 2015-2017. PLoS Biol. 2018;16(11):e2006930.

68. Börnigen D, Morgan XC, Franzosa EA, Ren B, Xavier RJ, Garrett WS, et al. Functional profiling of the gut microbiome in disease-associated inflammation. Genome Med. 2013;5(7):65.

69. Nguyen TL, Vieira-Silva S, Liston A, Raes J. How informative is the mouse for human gut microbiota research? Dis Model Mech. 2015;8(1):1-16.

70. Christensen L, Roager HM, Astrup A, Hjorth MF. Microbial enterotypes in personalized nutrition and obesity management. Am J Clin Nutr. 2018; 108(4):645-51. 
71. Hadrich D. Microbiome research is becoming the key to better understanding health and nutrition. Front Genet. 2018;9:212.

72. Tucker KL. Assessment of usual dietary intake in population studies of genediet interaction. Nutr Metab Cardiovasc Dis. 2007;17(2):74-81.

73. Marles RJ. Mineral nutrient composition of vegetables, fruits and grains: the context of reports of apparent historical declines. J Food Compos Anal. 2017;56:93-103.

74. Zhang L, Geelen A, Boshuizen HC, Ferreira J, Ocké MC. Importance of details in food descriptions in estimating population nutrient intake distributions. Nutr J. 2019;18(1):17

75. Schakel SF, Buzzard IM, Gebhardt SE. Procedures for estimating nutrient values for food composition databases. J Food Compos Anal. 1997;10(2): 102-14.

76. Ercolini D, Fogliano V. Food design to feed the human gut microbiota. J Agric Food Chem. 2018;66(15):3754-8

77. Maki KC, Slavin JL, Rains TM, Kris-Etherton PM. Limitations of observational evidence: implications for evidence-based dietary recommendations. Adv Nutr (Bethesda, Md). 2014;5(1):7-15.

78. Trepanowski JF, loannidis JPA. Perspective: limiting dependence on nonrandomized studies and improving randomized trials in human nutrition research: why and how. Adv Nutr. 2018;9(4):367-77.

79. Perez-Gregorio R, Simal-Gandara J. A critical review of bioactive food components, and of their functional mechanisms, biological effects and health outcomes. Curr Pharm Des. 2017;23(19):2731-41.

80. Lam YY, Maguire S, Palacios T, Caterson ID. Are the gut bacteria telling us to eat or not to eat? Reviewing the role of gut microbiota in the etiology, disease progression and treatment of eating disorders. Nutrients. 2017;9(6).

81. Davy KP, Davy BM. Advances in nutrition science and integrative physiology: insights from controlled feeding studies. Front Physiol. 2019;10: 1341.

82. Gibson TM, Ferrucci LM, Tangrea JA, Schatzkin A. Epidemiological and clinical studies of nutrition. Semin Oncol. 2010;37(3):282-96.

83. Hébert JR, Frongillo EA, Adams SA, Turner-McGrievy GM, Hurley TG, Miller $D R$, et al. Perspective: randomized controlled rials are not a panacea for diet-related research. Adv Nutr (Bethesda, Md). 2016;7(3):423-32.

84. Costea PI, Hildebrand F, Arumugam M, Bäckhed F, Blaser MJ, Bushman FD, et al. Enterotypes in the landscape of gut microbial community composition. Nat Microbiol. 2018;3(1):8-16.

85. de Moraes ACF, Fernandes GR, da Silva IT, Almeida-Pititto B, Gomes EP, Pereira ADC, et al. Enterotype may drive the dietary-associated cardiometabolic risk factors. Front Cell Infect Microbiol. 2017;7:47.

86. Gu Y, Wang X, Li J, Zhang Y, Zhong H, Liu R, et al. Analyses of gut microbiota and plasma bile acids enable stratification of patients for antidiabetic treatment. Nat Commun. 2017;8(1):1785

87. Zhong H, Penders J, Shi Z, Ren H, Cai K, Fang C, et al. Impact of early events and lifestyle on the gut microbiota and metabolic phenotypes in young school-age children. Microbiome. 2019;7(1):2.

88. Tebani A, Bekri S. Paving the way to precision nutrition through metabolomics. Front Nutr. 2019:6:41.

89. DIETary Assessment Tools NETwork. Available from: https://www.nutritools.org.

90. Hooson J, Hutchinson J, Warthon-Medina M, Hancock N, Greathead K, Knowles B, et al. A systematic review of reviews identifying UK validated dietary assessment tools for inclusion on an interactive guided website for researchers: www.nutritools.org. Critical Reviews in Food Science and Nutrition. 2019:1-25

91. Cade JE, Warthon-Medina M, Albar S, Alwan NA, Ness A, Roe M, et al. DIET@NET: best practice guidelines for dietary assessment in health research. BMC Med. 2017;15(1):202.

92. Eldridge A, Piernas C, Illner A-K, Gibney M, Gurinović M, de Vries J, et al. Evaluation of new technology-based tools for dietary intake assessment - an ILSI Europe Dietary Intake and Exposure Task Force evaluation. Nutrients. 2018;11(1):55

93. Timon CM, Evans K, Kehoe L, Blain RJ, Flynn A, Gibney ER, et al. Comparison of a web-based 24-h dietary recall tool (Foodbook24) to an interviewer-led 24-h dietary recall. Nutrients. 2017;9(5).

94. Holmes B, Dick K, Nelson M. A comparison of four dietary assessment methods in materially deprived households in England. Public Health Nutr. 2008;11(5):444-56.

95. Jackson KA, Byrne NM, Magarey AM, Hills AP. Minimizing random error in dietary intakes assessed by $24-h$ recall, in overweight and obese adults. Eur J Clin Nutr. 2008;62(4):537-43.
96. Tapsell LC, Neale EP, Satija A, Hu FB. Foods, nutrients, and dietary patterns: interconnections and implications for dietary guidelines. Adv Nutr (Bethesda, Md). 2016;7(3):445-54.

97. Zhang Z, Li D. Thermal processing of food reduces gut microbiota diversity of the host and triggers adaptation of the microbiota: evidence from two vertebrates. Microbiome. 2018;6(1):99.

98. Carmody RN, Bisanz JE, Bowen BP, Maurice CF, Lyalina S, Louie KB, et al. Cooking shapes the structure and function of the gut microbiome. Nat Microbiol. 2019:4(12):2052-63.

99. Wang Z, Bergeron N, Levison BS, Li XS, Chiu S, Jia X, et al. Impact of chronic dietary red meat, white meat, or non-meat protein on trimethylamine $\mathrm{N}$ oxide metabolism and renal excretion in healthy men and women. Eur Heart J. 2019:40(7):583-94.

100. Hooton F, Menichetti G, Barabási A-L. FoodMine: exploring food contents in scientific literature. bioRxiv. 2019; 2019.12.17.880062.

101. Zhou L, Zhang C, Liu F, Qiu Z, He Y. Application of deep learning in food: a review. Compr Rev Food Sci Food Saf. 2019;18(6):1793-811.

102. Neyrinck AM, Rodriguez J, Vinoy S, Maquet V, Walter J, Bischoff SC, et al. The FiberTAG project: tagging dietary fibre intake by measuring biomarkers related to the gut microbiota and their interest for health. Nutr Bull. 2020; 45(1):59-65.

103. Le Roy Cl, Bowyer RCE, Castillo-Fernandez JE, Pallister T, Menni C, Steves CJ, et al. Dissecting the role of the gut microbiota and diet on visceral fat mass accumulation. Sci Rep. 2019;9(1):9758.

104. Le Roy Cl, Wells PM, Si J, Raes J, Bell JT, Spector TD. Red wine consumption associated with increased gut microbiota a-diversity in 3 independent cohorts. Gastroenterology. 158(1):270-2(e2).

105. Lachat C, Hawwash D, Ocké MC, Berg C, Forsum E, Hörnell A, et al. Strengthening the reporting of observational studies in epidemiology-nutritional epidemiology (STROBE-nut): an extension of the STROBE statement. PLoS Med. 2016;13(6):e1002036.

106. Louca P, Menni C, Padmanabhan S. Genomic determinants of hypertension with a focus on metabolomics and the gut microbiome. Am J Hypertens. 2020;33(6):473-81. https://doi.org/10.1093/ajh/hpaa022.

107. Forgie AJ, Fouhse JM, Willing BP. Diet-microbe-host interactions that affect gut mucosal integrity and infection resistance. Front Immunol. 2019;10:1802.

108. De Filippis F, Pellegrini N, Vannini L, Jeffery IB, La Storia A, Laghi L, et al. High-level adherence to a Mediterranean diet beneficially impacts the gut microbiota and associated metabolome. Gut. 2016;65(11):1812.

109. Read MN, Holmes AJ. Towards an integrative understanding of diet-hostgut microbiome interactions. Front Immunol. 2017:8:538.

110. Cani PD. Human gut microbiome: hopes, threats and promises. Gut. 2018; 67(9):1716.

111. Wu GD, Compher C, Chen EZ, Smith SA, Shah RD, Bittinger $K$, et al. Comparative metabolomics in vegans and omnivores reveal constraints on diet-dependent gut microbiota metabolite production. Gut. 2016; 65(1):63-72.

112. Marcobal A, Yusufaly T, Higginbottom S, Snyder M, Sonnenburg JL, Mias Gl. Metabolome progression during early gut microbial colonization of gnotobiotic mice. Sci Rep. 2015;5(1):11589.

113. Kok MGM, Ruijken MMA, Swann JR, Wilson ID, Somsen GW, de Jong GJ. Anionic metabolic profiling of urine from antibiotic-treated rats by capillary electrophoresis-mass spectrometry. Anal Bioanal Chem. 2013;405(8):2585-94.

114. Le Roy Cl, Woodward MJ, Ellis RJ, La Ragione RM, Claus SP. Antibiotic treatment triggers gut dysbiosis and modulates metabolism in a chicken model of gastro-intestinal infection. BMC Vet Res. 2019;15(1):37.

115. Rowland I, Gibson G, Heinken A, Scott K, Swann J, Thiele I, et al. Gut microbiota functions: metabolism of nutrients and other food components. Eur J Nutr. 2018:57(1):1-24

116. Gotoh A, Katoh T, Sakanaka M, Ling Y, Yamada C, Asakuma S, et al. Sharing of human milk oligosaccharides degradants within bifidobacterial communities in faecal cultures supplemented with Bifidobacterium bifidum. Sci Rep. 2018;8(1):13958

117. Soto-Martin EC, Warnke I, Farquharson FM, Christodoulou M, Horgan G, Derrien $\mathrm{M}$, et al. Vitamin biosynthesis by human gut butyrate-producing bacteria and cross-feeding in synthetic microbial communities. mBio. 2020; 11(4):e00886-20.

118. Gurwara S, Ajami NJ, Jang A, Hessel FC, Chen L, Plew S, et al. Dietary nutrients involved in one-carbon metabolism and colonic mucosaassociated gut microbiome in individuals with an endoscopically normal colon. Nutrients. 2019;11(3). 
119. Steinert RE, Lee Y-K, Sybesma W. Vitamins for the gut microbiome. Trends Mol Med. 2020;26(2):137-40.

120. Sinha R, Ahsan H, Blaser M, Caporaso JG, Carmical JR, Chan AT, et al. Next steps in studying the human microbiome and health in prospective studies, Bethesda, MD, May 16-17, 2017. Microbiome. 2018;6(1):210.

121. Hughes RL, Marco ML, Hughes JP, Keim NL, Kable ME. The role of the gut microbiome in predicting response to diet and the development of precision nutrition models - part l: overview of current methods. Adv Nutr. 2019;10(6):953-78.

122. Moola S, Munn Z, Tufanaru C, Aromataris E, Sears K, Sfetcu R, Currie M, Qureshi R, Mattis P, Lisy K, Mu P-F. Chapter 7: Systematic reviews of etiology and risk. In: Joanna Briggs Institue Reviewer's Manual [Internet]: The Joanna Briggs Institute; 2017. Available from: https://reviewersmanual.joannabriggs. org/.

123. Geva-Zatorsky N, Alvarez D, Hudak JE, Reading NC, Erturk-Hasdemir D, Dasgupta S, et al. In vivo imaging and tracking of host-microbiota interactions via metabolic labeling of gut anaerobic bacteria. Nat Med. 2015 21(9):1091-100.

124. Peters DPC, Havstad KM, Cushing J, Tweedie C, Fuentes O, VillanuevaRosales N. Harnessing the power of big data: infusing the scientific method with machine learning to transform ecology. Ecosphere. 2014;5(6):art67.

125. Qu K, Guo F, Liu X, Lin Y, Zou Q. Application of machine learning in microbiology. Front Microbiol. 2019;10:827.

126. Trang Tran TN, Atas M, Felfernig A, Stettinger M. An overview of recommender systems in the healthy food domain. J Intell Inf Syst. 2018; 50(3):501-26.

127. Zeevi D, Korem T, Zmora N, Israeli D, Rothschild D, Weinberger A, et al. Personalized nutrition by prediction of glycemic responses. Cell. 2015;163(5): 1079-94.

128. Shilo S, Rossman H, Segal E. Axes of a revolution: challenges and promises of big data in healthcare. Nat Med. 2020;26(1):29-38.

129. Awany D, Allali I, Dalvie S, Hemmings S, Mwaikono KS, Thomford NE, et al. Host and microbiome genome-wide association studies: current state and challenges. Front Genet. 2019;9:637.

130. Duvallet C. Meta-analysis generates and prioritizes hypotheses for translational microbiome research. Microb Biotechnol. 2018;11(2):273-6.

131. Doupe P, Faghmous J, Basu S. Machine learning for health services researchers. Value Health. 2019;22(7):808-15.

\section{Publisher's Note}

Springer Nature remains neutral with regard to jurisdictional claims in published maps and institutional affiliations. 